\title{
Is Magnetic Resonance Imaging Diffusion Restriction of the Optic Disc Head a New Marker for Idiopathic Intracranial Hypertension?
}

\author{
Elanagan Nagarajan ${ }^{1} \quad$ Lakshmi P. Digala ${ }^{2}$ Manjamalai Sivaraman ${ }^{2}$ Pradeep C. Bollu² \\ ${ }^{1}$ Department of Neurology, Columbia University, New York, New York, \\ Address for correspondence Elanagan Nagarajan, MD, Department \\ United States \\ 2Department of Neurology, University of Missouri, Columbia, \\ Missouri, United States \\ of Neurology, Columbia University, New York, 710 West 168th Street, \\ NY 10032, United States (e-mail: en2452@cumc.columbia.edu).
}

J Neurosci Rural Pract 2020;11:170-174

\begin{abstract}
Background Idiopathic intracranial hypertension (IIH) is a headache syndrome due to raised intracranial pressure of unknown etiology. Before making the diagnosis of $\mathrm{IIH}$, secondary causes of raised intracranial pressure must be ruled out. The radiological features associated with this condition have variable sensitivity and specificity. In this case series, we aim to describe a potential new radiological marker of $\mathrm{IH}$, that is, diffusion restriction, in the optic disc head and propose that this can be a specific finding in the appropriate clinical picture.

Importance IIH causes vision loss and disabling daily headaches. The diagnosis of this condition is based on history and physical examination findings. Magnetic resonance imaging (MRI) is used to exclude other causes, but specific radiological markers for the diagnosis of $\mathrm{IIH}$ are lacking.

Observations Five patients presented with the main complaint of headache, which was associated with blurry vision. All of our patients had a formal neuro-ophthalmological evaluation that confirmed the presence of optic disc edema in both eyes. They also underwent an MRI of the brain that showed diffusion restriction in the optic nerve head in either eye or both eyes. Patients underwent lumbar puncture in the lateral

Keywords

- idiopathic intracranial hypertension

- pseudotumor cerebri

- papilledema

- diffusion restriction decubitus position, which revealed cerebrospinal fluid opening pressures $>25 \mathrm{~cm} \mathrm{H}_{2} \mathrm{O}$. They all responded well to standard treatments, with the resolution of symptoms in their follow-up appointments.

Conclusion and Relevance The MRI diffusion restriction in the optic nerve head may be a reliable noninvasive marker for the diagnosis of $\mathrm{IH}$ in the appropriate clinical picture.
\end{abstract}

\section{Introduction}

Headaches are one of the leading causes of both outpatient and emergency room visits and are an important public health problem, particularly among women during their reproductive years. Migraine is the most common headache disorder in this age group followed by idiopathic intracranial hypertension (IIH). ${ }^{1}$ IIH is a well-characterized headache syndrome caused by elevated intracranial pressure
(ICP) of unknown etiology. It causes significant morbidity in untreated cases, including permanent vision loss, and therefore early diagnosis and intervention is warranted. ${ }^{2}$ Magnetic resonance imaging (MRI) is the imaging modality of choice to rule out other potential causes of raised ICP. Common neuroimaging findings in patients with IIH include empty sella, flattening of the posterior globes, distention of the optic nerve sheaths (ONSs), cerebellar tonsillar herniation, meningoceles, and transverse venous sinus stenosis. ${ }^{3}$ All these 
reported MRI findings have high sensitivity and specificity in diagnosing patients with $\mathrm{IIH}^{3-5}$

In this case series, we present five patients with $\mathrm{IIH}$ who had diffusion restriction in one or both optic discs with a corresponding apparent diffusion coefficient (ADC) correlate.

\section{Methods}

The study was reviewed and approved by the Institutional Review Board of the University of Missouri Hospital.

\section{Case 1}

A 29-year-old Hispanic female presented to our emergency department with a 5-day history of headache. The headache was associated with blurry vision, nausea, and vomiting. She was on tacrolimus because of focal segmental glomerular sclerosis. She was evaluated by a neuro-ophthalmologist and noted to have grade 2 optic disc edema in the left eye and grade 5 optic disc edema in the right eye along with subtle hemorrhage. The MRI of her brain showed edema and restricted diffusion in the optic papillae bilaterally, suggestive of ischemic changes ( - Fig. 1). There were no other abnormal findings on MRI of the brain. Magnetic resonance (MR) venogram did not show any major vessel occlusion or stenosis. Lumbar puncture showed an opening pressure of $50 \mathrm{~cm} \mathrm{H}_{2} \mathrm{O}$. These findings in the context of her clinical presentation and examination were consistent with IIH. She was started on acetazolamide, and tacrolimus was stopped. Her headache improved significantly by the time of discharge.

\section{Case 2}

A 20-year-old Caucasian female with no significant past medical history, except for recent steady weight gain, presented to our ophthalmology clinic for an evaluation of occasional headaches. These headaches were associated with pulsating tinnitus and occasional pressure in the back of her eye. The headaches were not associated with photophobia, nausea, emesis or, alteration by positional changes. Funduscopic examination showed grade 2 optic disc edema in the right eye and grade 3 optic disc edema in the left eye. Humphrey's visual field (HVF) testing showed enlargement of blind spots in both eyes and nasal scotoma in the left eye.

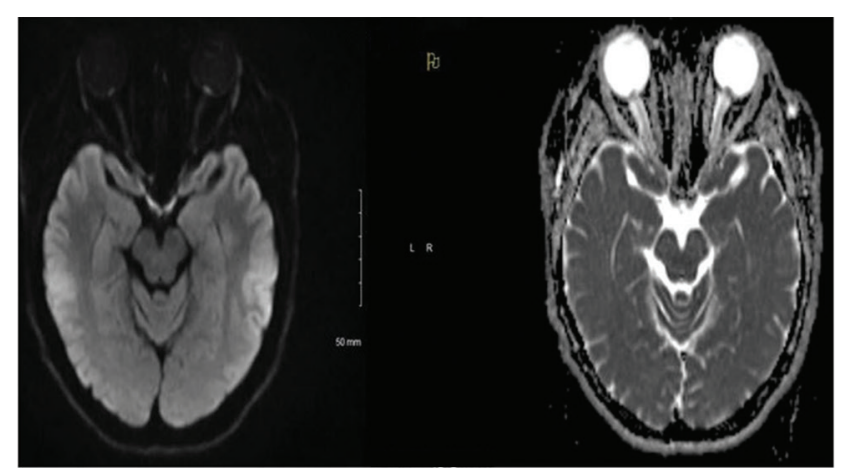

Fig. 1 MRI (magnetic resonance imaging) of the brain showing edema and restricted diffusion in the optic papillae bilaterally, suggestive of ischemic changes.
The MRI of the brain showed edema and restricted diffusion in the optic papillae bilaterally, more pronounced in the right eye ( - Fig. 2). The papillae also demonstrated mild postcontrast enhancement. MR venogram showed a hypoplastic left transverse sinus, but no significant vessel stenosis or occlusion was noted. Lumbar puncture showed an elevated opening pressure of $35 \mathrm{~cm} \mathrm{H}_{2} 0$. The patient was started on acetazolamide. Her subsequent clinic visit showed improvement in papilledema (grade 1 in the right eye and grade 2 in the left eye). HVF testing also showed improvement of blind spots in both eyes and nasal scotoma in the left eye that has improved from the previous clinic visit.

\section{Case 3}

A26-year-old Caucasian female presented to our emergency department with a 5-day history of intermittent binocular diplopia and headache. She complained of retro-orbital pain that was more pronounced in the left eye with occasional floaters. She was noted to have a weight gain of 10 pounds over the past 6 months. Funduscopic examination revealed bilateral optic disc edema (grade 2 in the left eye and grade 1 in the right eye) along with mild left lateral rectus palsy. The MRI of her brain showed edema, restricted diffusion, and postcontrast enhancement in the optic papillae bilaterally that was more pronounced on the

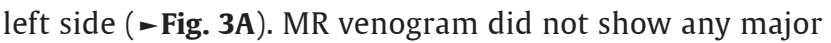
vessel occlusion or stenosis. Lumbar puncture showed an elevated opening pressure of $37 \mathrm{~cm} \mathrm{H}_{2} \mathrm{O}$. HVF showed peripheral scotoma on the right and dense central scotoma on the left. The diagnosis of IIH was made and the patient was started on acetazolamide. The intensity and frequency of headaches improved at her subsequent clinical visit. But the patient was not able to tolerate the acetazolamide due to nausea, and subsequently a lumboperitoneal shunt was placed. The repeat MRI after the shunt placement did not show any evidence of edema of the optic disc ( - Fig. 3B).

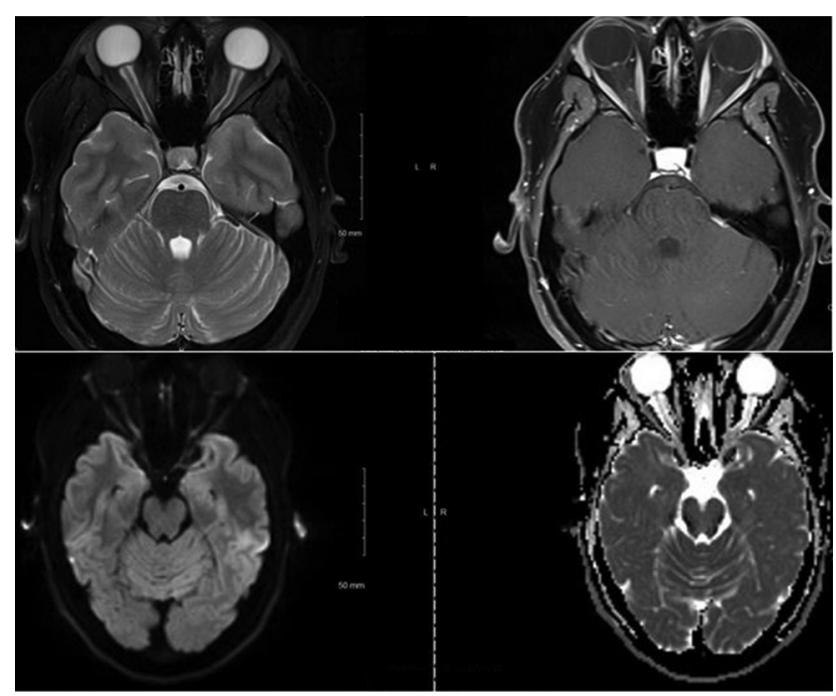

Fig. 2 MRI (magnetic resonance imaging) of the brain showing edema and restricted diffusion in the optic papillae bilaterally, which is more pronounced in the right eye. 

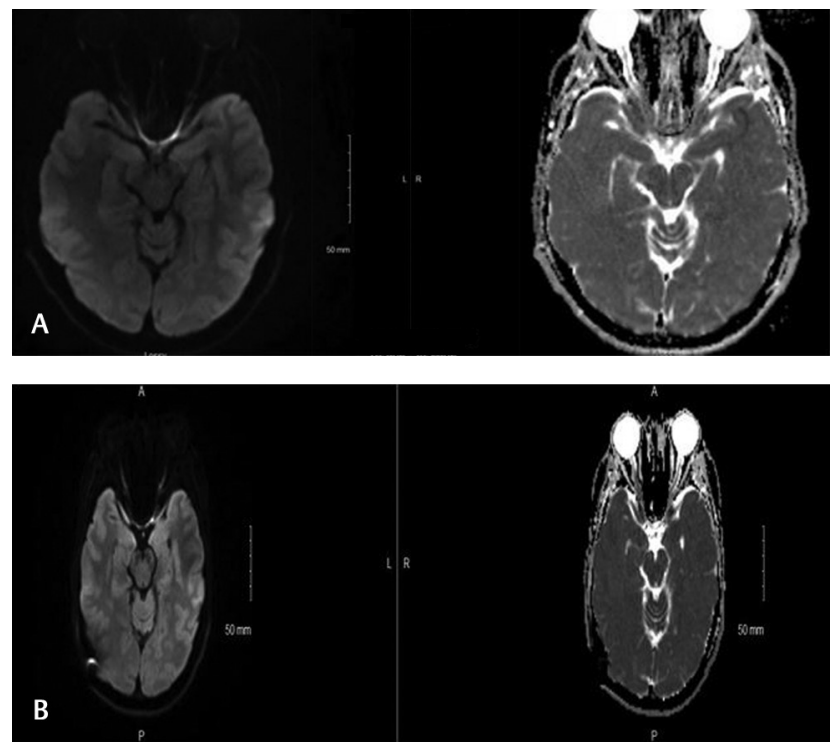

Fig. 3 (A) MRI (magnetic resonance imaging) of the brain showing edema, restricted diffusion, and postcontrast enhancement in the optic papillae bilaterally that was more pronounced in the left eye. (B) The repeat MRI (magnetic resonance imaging) after the shunt placement did not show any evidence of edema of the optic disc.

\section{Case 4}

A 26-year-old Caucasian male presented to the hospital with a 3-week history of blurry vision in both eyes. His vision was worse in the right eye and was associated with dark spots and a mild headache. He reported a weight gain of 20 pounds in the past 6 months. Funduscopic examination revealed grade 4 optic disc edema in the right eye and grade 3 in the left eye with peripapillary hemorrhage. MRI of his brain with contrast showed edema and restricted diffusion in the optic papillae bilaterally, significantly more so in the right eye than the left eye (-Fig. 4). Venous sinuses did not show any abnormality. Lumbar puncture revealed an opening pressure of $55 \mathrm{~cm} \mathrm{H}_{2} 0$. He was started on acetazolamide. During the follow-up, the patient had worsening of headache and blurry vision and therefore underwent optic nerve sheath (ONS) fenestration.

\section{Case 5}

A 23-year-old right-handed woman presented with a 4-day history of blurry vision. She describes her visual disturbance as a painful blurring in both eyes (greater in the right eye) and double vision along with seeing flashes of light and floaters. She also had vertical gaze paresis. The patient was evaluated by a neuro-ophthalmologist and was found to have a grade 4 papilledema bilaterally. HVF showed constriction of peripheral visual field in both eyes (greater in the right eye). MRI of the brain with contrast showed edema and diffusion restriction in the bilateral optic papillae, more significantly in the right eye (-Fig. 5). Lumbar puncture was performed that showed an elevated opening pressure of $>55 \mathrm{~cm} \mathrm{H}_{2} \mathrm{O}$. Her headache did not improve with medical management, and the patient eventually underwent ONS fenestration.

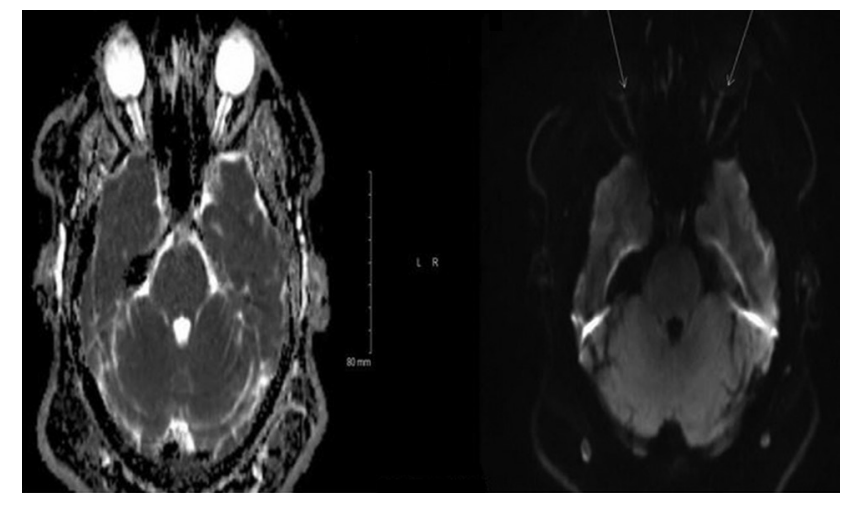

Fig. $4 \mathrm{MRI}$ (magnetic resonance imaging) of the brain with contrast showing edema and restricted diffusion in the optic papillae bilaterally, significantly more so in the right eye than the left eye.

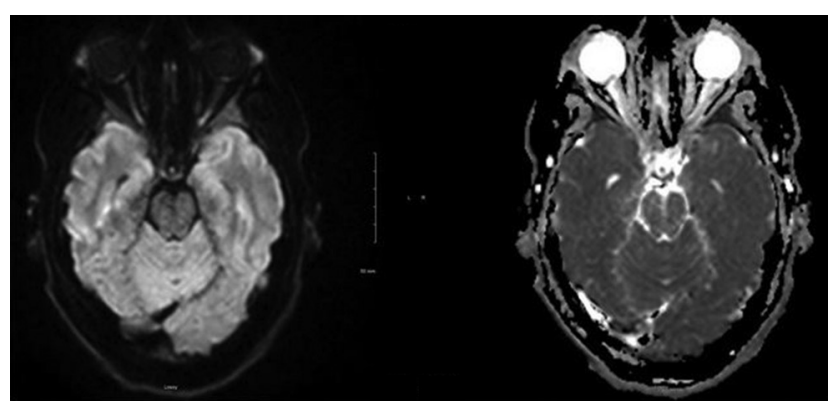

Fig. $5 \mathrm{MRI}$ (magnetic resonance imaging) of the brain with contrast showing edema and diffusion restriction in the bilateral optic papillae, more significantly in the right eye.

\section{Discussion}

Papilledema refers to optic disc swelling because of raised ICP. ${ }^{3}$ MRI is the commonly used noninvasive diagnostic modality to study the optic nerves and other orbital structures. $^{3}$ Common radiological signs associated with $\mathrm{IIH}$ are given in - Table $\mathbf{1}^{3}$ Brodsky and Vaphiades ${ }^{3}$ proposed that the presence of posterior scleral flattening and prelaminar enhancement on imaging is a confirmatory finding of IIH in the presence of papilledema. ${ }^{6}$ The other radiological signs that are associated with IIH, however, are nonspecific. ${ }^{3-6}$ In our study, we present high T2-weighted signal changes in the optic disc head along with restriction of diffusion as a potential new radiological marker in cases of $\mathrm{IIH}$, and this finding suggests optic nerve ischemia in these patients.

The sensitivity of transverse sinus stenosis was reported to be approximately $90 \%$ in patients with $\mathrm{IIH}^{7}{ }^{7}$ Three patients in our case series had a dedicated imaging for the venous system. Among them, one patient had hypoplastic left transverse sinus, and the venous system was unremarkable in all other patients. Two patients did not have venogram, but MRI of the brain with contrast did not show any suggestion of venous sinus occlusion. Sari et al reported that MRI of the brain with contrast is highly sensitive for studying the venous system when compared with MR venogram and noncontrast imaging of the brain. ${ }^{7}$

All the patients in our case series underwent lumbar puncture, and the opening pressure was $>25 \mathrm{~mm} \mathrm{H}_{2} \mathrm{O}$. These 
Table 1 Common radiological signs with IIH

\begin{tabular}{|l|l|l|}
\hline Imaging findings & $\begin{array}{l}\text { Sensitivity } \\
(\%)\end{array}$ & $\begin{array}{l}\text { Specificity } \\
\text { (\%) }\end{array}$ \\
\hline Empty sella & 80 & 83 \\
\hline $\begin{array}{l}\text { Meningocele/meningoen- } \\
\text { cephalocele }\end{array}$ & 11 & 0 \\
\hline Foramen ovale widening & 50 & 80 \\
\hline $\begin{array}{l}\text { Posterior flattening of the } \\
\text { posterior poles }\end{array}$ & 66 & 98 \\
\hline Optic nerve head protrusion & 36 & 99 \\
\hline $\begin{array}{l}\text { Distention of the optic nerve } \\
\text { sheath }\end{array}$ & 58 & 89 \\
\hline $\begin{array}{l}\text { Vertical tortuosity of the } \\
\text { optic nerve }\end{array}$ & 43 & 90 \\
\hline Transverse sinus stenosis & 97 & 93 \\
\hline $\begin{array}{l}\text { Enhancement of the optic } \\
\text { nerve head }\end{array}$ & 17 & 99 \\
\hline Tonsillar herniation > 5 mm & 16 & 95 \\
\hline
\end{tabular}

findings are concordant with the opening pressure reported in patients with IIH for the diagnosis. ${ }^{8}$

The first case of IIH was reported in the 1890s by a German physician Heinrich Quincke, who called it "serous meningitis." Subsequently, Nonne and Dandy reported similar case series of increased ICP without tumor and coined the name "pseudotumor cerebri." 10,11

The reported incidence of IIH in the general population is between 1 and 3 per 100,000 per year, with a higher incidence in females (up to 12 to 28 per 100,000 per year) during their reproductive years. ${ }^{12-14} \mathrm{Clinical}$ features of this condition include headache, transient visual obscurations, diplopia, and pulsatile tinnitus. In untreated cases, vision loss is a major complication and sometimes can be very profound within a few days. In a recent study from the United Kingdom, the annual incidence of blindness in patients with IIH is 1 to $2 \%{ }^{15}$ Friedman proposed diagnostic criteria for IIH that include both patients with and those without papilledema, ${ }^{16}$ and all of the patients in this case series fulfill those diagnostic criteria. The management of these patients is based on preserving the visual function along with reducing the disability from headache. The treatment options include weight loss in conjunction with medications such as acetazolamide, topiramate, furosemide, and, sometimes, corticosteroids. If the medical therapy fails, the surgical options include serial lumbar punctures, ONS fenestration, ventriculoperitoneal shunt, lumboperitoneal shunt, and transverse sinus stenting. Studies have shown that surgical interventions are effective in improving visual symptoms, preventing vision loss and reducing the morbidity associated with headache., ${ }^{9,15,16}$

The pathophysiology of IIH and papilledema is still controversial, and the exact mechanism is not clear. The subarachnoid space underneath the ONS is in direct anatomical connection with cerebrospinal fluid (CSF) surrounding the brain. Increased ICP causes a direct transmission of this pressure, resulting in distention of the ONS, as shown in -Fig. $\mathbf{6 A}$, B, The increase in ICP causes an enlargement of the ONS diameter and changes in tortuosity. This process eventually leads to alteration in the
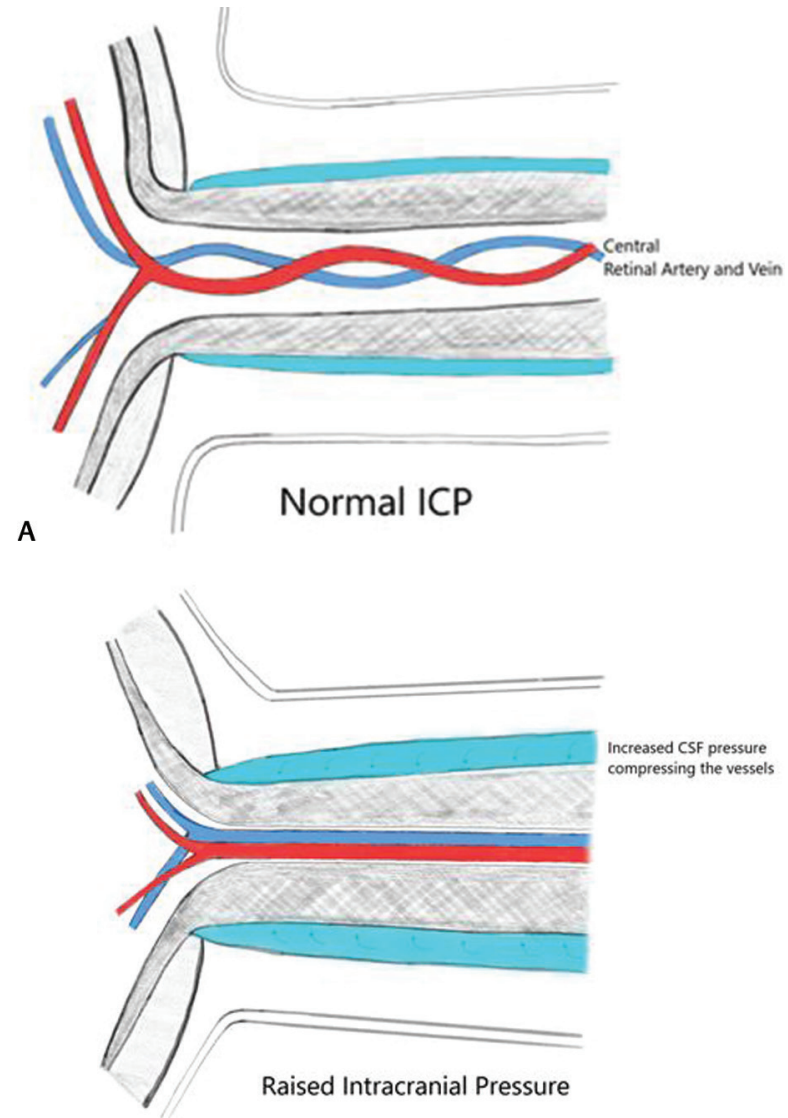

B

Fig. 6 (A) Anatomy of the optic nerve and the central retinal artery and vein present in the center at normal pressure. (B) Compression of both the central retinal artery and vein during raised intracranial pressure.

metabolic processes of the nerve and subsequent edema, ischemia, and, finally, visual impairment or loss.,17 $\mathrm{T} 2$-weighted images are used for the analysis of orbital structures and the optic sheath..$^{18}$ Most of the optic nerve findings are reported from thin-section or volumetric fat-saturated T2-weighted images of the orbit. ${ }^{19}$ In our series, in addition to all reported findings from the literature, we found increased T2-weighted signal changes in the optic disc head and diffusion restriction. We propose that continued pressure on the optic nerves would result in ischemic insult in the optic nerve head. In ischemic stroke, diffusion sequence on brain MRI shows increased signal with a low ADC signal, and these are the same MRI findings in our case series. To our knowledge, MRI findings involving optic disc heads have not been described in IIH. Given the paucity of specific radiological features in cases of IIH, we propose that the diffusion restriction in the optic disc heads in a patient with clinically suggestive IIH may be considered a fairly specific finding.

\section{Conclusion}

IIH is a headache syndrome that can result in visual deficits including permanent vision loss if left untreated. Diagnosis of this condition relies on proper clinical history and physical examination findings along with measurement of CSF 
pressure. The finding of a unilateral or bilateral MR diffusion restriction of optical heads in $\mathrm{IIH}$ patients has the potential to become a new IIH diagnostic marker if it is confirmed in larger and controlled series. We propose that diffusion restriction in the optic disc heads in the appropriate clinical picture may be a specific radiological finding of IIH.

\section{Note}

This study was approved by the Institutional Board Review of the University of Missouri Health Care- Columbia.

\section{Funding}

None.

\section{Conflict of Interest}

None declared.

\section{References}

1 Burch RC, Loder S, Loder E, Smitherman TA. The prevalence and burden of migraine and severe headache in the United States: updated statistics from government health surveillance studies. Headache 2015;55(1):21-34

2 Jensen RH, Radojicic A, Yri H. The diagnosis and management of idiopathic intracranial hypertension and the associated headache. Ther Adv Neurol Disorder 2016;9(4):317-326

3 Bidot S, Saindane AM, Peragallo JH, Bruce BB, Newman NJ, Biousse V. Brain imaging in idiopathic intracranial hypertension. J Neuroophthalmol 2015;35(4):400-411

4 Lim MJ, Pushparajah K, Jan W, Calver D, Lin JP. Magnetic resonance imaging changes in idiopathic intracranial hypertension in children. J Child Neurol 2010;25(3):294-299

5 Agid R, Farb RI, Willinsky RA, Mikulis DJ, Tomlinson G. Idiopathic intracranial hypertension: the validity of cross-sectional neuroimaging signs. Neuroradiology 2006;48(8):521-527

6 Brodsky MC, Vaphiades M. Magnetic resonance imaging in pseudotumorcerebri.Ophthalmology 1998;105(9):1686-1693

7 Sari S, Verim S, Hamcan S, et al. MRI diagnosis of dural sinus - cortical venous thrombosis: immediate post-contrast
3D GRE T1-weighted imaging versus unenhanced MR venography and conventional MR sequences. Clin Neurol Neurosurg 2015;134:44-54

8 Corbett JJ, Mehta MP. Cerebrospinal fluid pressure in normal obese subjects and patients with pseudotumor cerebri. Neurology 1983;33(10):1386-1388

9 Mollan SP, Ali F, Hassan-Smith G, Botfield H, Friedman DI, Sinclair AJ. Evolving evidence in adult idiopathic intracranial hypertension: pathophysiology and management. J Neurol Neurosurg Psychiatry 2016;87(9):982-992

10 Nonne M. Über Fälle vom Symptomkomplex 'tumor cerebri' mit Ausgang in Heilung (Pseudotumor cerebri). Über Letal Verlaufene Fälle von 'Pseudotumor Cerebri' mit Sektionsbefund. Dtsch Z Nervenheilkd 1904;27:169-216

11 Dandy WE. Intracranial pressure without brain tumor: diagnosis and treatment. Ann Surg 1937;106(4):492-513

12 Radhakrishnan K, Thacker AK, Bohlaga NH, Maloo JC, Gerryo SE. Epidemiology of idiopathic intracranial hypertension: a prospective and case-control study. J Neurol Sci 1993;116(1):18-28

13 McCluskey G, Mulholland DA, McCarron P, McCarron MO. Idiopathic intracranial hypertension in the Northwest of Northern Ireland: epidemiology and clinical management. Neuroepidemiology 2015;45(1):34-39

14 Andrews LE, Liu GT, Ko MW. Idiopathic intracranial hypertension and obesity. Horm Res Paediatr 2014;81(4):217-225

15 Best J, Silvestri G, Burton B, Foot B, Acheson J. The incidence of blindness due to idiopathic intracranial hypertension in the UK. Open Ophthalmol J 2013;7:26-29

16 Friedman DI. The pseudotumor cerebri syndrome. Neurol Clin 2014;32(2):363-396

17 Sivasankar R, Pant R, Indrajit IK, et al. Imaging and interventions in idiopathic intracranial hypertension: a pictorial essay. Indian J Radiol Imaging 2015;25(4):439-444

18 Kimberly HH, Noble VE. Using MRI of the optic nerve sheath to detect elevated intracranial pressure. Crit Care 2008;12(5):181

19 Holbrook J, Saindane AM. Imaging of intracranial pressure disorders. Neurosurgery 2017;80(3):341-354 